\title{
Prognoses in Pathologically Confirmed T1 Lower Rectal Cancer Patients with or without Preoperative Therapy: An Analysis Using the Surveillance, Epidemiology, and End Results Database
}

\author{
Tetsuro Taira Hiroaki Nozawa Kazushige Kawai Kazuhito Sasaki \\ Koji Murono Shigenobu Emoto Junko Kishikawa Tsuyoshi Ozawa \\ Yuichiro Yokoyama Shinya Abe Yuzo Nagai Hiroyuki Anzai \\ Hirofumi Sonoda Soichiro Ishihara
}

Department of Surgical Oncology, The University of Tokyo, Tokyo, Japan

\section{Keywords}

Pathological T1 · Preoperative chemoradiotherapy ·

Prognosis · Rectal cancer · Surveillance, Epidemiology, and End Results

\footnotetext{
Abstract

Introduction: Preoperative chemoradiotherapy (CRT) is the standard therapy for downstaging in locally advanced lower rectal cancer. However, it remains unclear whether rectal cancers downstaged by preoperative therapy show similar prognoses to those of the same stage without preoperative therapy. We previously demonstrated that preoperative CRT did not affect prognosis of rectal cancer with pathological T1N0 (pT1N0) stage in a single institute. Here, using a larger dataset, we compared prognoses of (y)pT1 rectal cancer stratified by the use of preoperative therapy and analyzed prognostic factors. Methods: Cases of pT1N0 rectal cancer, registered between 2004 and 2016, were extracted from the Surveillance, Epidemiology, and End Results database. Pa-
}

tients were categorized as the "ypT1 group" if they had undergone preoperative therapy before surgery or as the "pT1 group" if they had undergone surgery alone. Overall survival (OS) and cancer-specific survival (CSS) between these groups of patients were compared. Factors associated with CSS and OS were identified by univariate and multivariate analyses. Results: Among 3,757 eligible patients, ypT1 and pT1 groups comprised 720 and 3,037 patients, respectively. While ypT1 patients showed poorer CSS than ypT1 patients, there was no significant difference in OS. Preoperative therapy was not an independent prognostic factor for CSS or OS. Multivariate analysis identified age and histological type as significant factors associated with CSS. Sex, age, race, and number of lymph nodes dissected were identified as significant factors associated with OS. Conclusions: Prognosis among patients with (y)p T1N0 rectal cancer was similar irrespective of whether they underwent preoperative therapy, which is consistent with our previous observations.

(c) 2021 The Author(s)

Published by S. Karger AG, Basel (c) 2021 The Author(s).

Published by S. Karger AG, Basel

This is an Open Access article licensed under the Creative Commons Attribution-NonCommercial-4.0 International License (CC BY-NC) (http://www.karger.com/Services/OpenAccessLicense), applicable to the online version of the article only. Usage and distribution for commercial purposes requires written permission. 


\section{Introduction}

Preoperative chemoradiotherapy (CRT) followed by total mesorectal excision (TME)-based surgery is one of the standard treatments for rectal cancer that penetrates the proper muscle layer, and this strategy contributes to reducing local recurrence rates [1]. Furthermore, patients who have achieved pathological complete response and downstaging show good oncological outcomes [2-5]. More recently, preoperative chemotherapy and total neoadjuvant therapy have been introduced as treatments for lower rectal cancer [6-8]. However, it remains unclear whether rectal cancer downstaged by preoperative therapies has a different prognosis from that of the same stage without these treatments.

Only a few studies have compared the prognosis of stage I rectal cancer after preoperative CRT (ypT12N0M0) with treatment-naïve stage I rectal cancer (pT12N0M0) [9-13]. A previous study based on cases registered in the Surveillance, Epidemiology, and End Results (SEER) database between 2004 and 2012 demonstrated that ypStage I rectal cancer patients $(n=2,240)$ had shorter overall survival (OS) and cancer-specific survival (CSS) than pStage I patients $(n=8,433)[13]$. However, their study contained an obvious bias in the number of (y)pT1 and (y)pT2 patients with and without preoperative CRT, and this predominance might have contributed to reduced survival in patients receiving CRT. In contrast, we previously conducted a single-center study that included only patients with lower rectal cancer with a pathological T1 stage, demonstrating that there was no difference in relapse-free survival (RFS) between ypT1 and pT1 patients [14]. One of the limitations of that study was a small sample size (86 patients). Therefore, this study aimed to analyze data from a larger number of rectal cancer patients using the SEER database to verify our previous findings.

\section{Materials and Methods}

Patient Selection from the SEER Database

Cases of rectal cancer (C20.9 Rectum, NOS) between 2004 and 2016 were extracted from the SEER database (SEER*Stat 8.3.8) according to Site Recode classification. Surgery and radiation sequences were limited to "radiation prior to surgery," "radiation before and after surgery," "surgery both before and after radiation," and "no radiation and/or cancer-directed surgery." Surgery and systemic therapy sequences were limited to "systemic therapy before surgery," "systemic therapy after surgery," "systemic therapy both before and after surgery," and "no systemic therapy and/or surgical procedures." Histological types were lim- ited to adenocarcinoma (ICD-03, 8140/3, 8210/3, 8261/3, $8263 / 3$ ), mucinous adenocarcinoma (ICD-03, 8480/3), and signet ring cell carcinoma (ICD-03, 8490/3). We only reviewed patients who had pathologically confirmed $\mathrm{T} 1$ rectal cancer without lymph node and/or distant metastases. Patients with unknown number of lymph nodes retrieval, with unknown treatment sequence, and with unknown TNM stage were excluded. Data on the following variables were collected: sex, age at diagnosis, race, histological type, pathological grading, and the number of lymph nodes dissected.

\section{Patient Classification}

In this study, patients who underwent preoperative therapy and TME-based surgery were classified as the "ypT1 group," whereas those treated by surgery alone were classified as the "pT1 group."

\section{Statistical Analyses}

Categorical variables were compared using the $\chi^{2}$ test, while continuous variables were compared using the unpaired $t$ test. Factors associated with CSS and OS were estimated by univariate and multivariate analyses using the Cox proportional hazards model, where continuous variables were dichotomized by their median or mean values, except for the variable "number of lymph nodes dissected (12)." Only variables with $p<0.05$ in univariate analysis were subjected to multivariate analysis. CSS and OS curves were estimated using the Kaplan-Meier method and compared using the log-rank test. All analyses were performed using the JMP Pro 15.1 software (SAS Institute Inc., Cary, NC, USA); $p<0.05$ was considered significant.

\section{Results}

\section{Patient Characteristics}

We extracted data on 3,757 eligible patients; of these, there were 720 patients in the ypT1 group and 3,037 patients in the pT1 group. Comparison of clinical and pathological characteristics between the ypT1 and the pT1 groups (Table 1) showed higher proportion of male ( $64 \%$ vs. $59 \% ; p=0.010)$ and black patients ( $11 \%$ vs. $7 \%$; $p<0.001)$ in the ypT1 group than in the $\mathrm{pT} 1$ group. $\mathrm{Pa}-$ tients in the ypT1 group were significantly younger than those in the pT1 group (average, 61.2 vs. $62.3 ; p=0.030$ ). There was a higher proportion of patients with well or moderate differentiated adenocarcinoma in the pT1 group than in the ypT1 group ( $83 \%$ vs. $76 \%$; $p<0.001)$. The number of lymph nodes retrieved was higher in the pT1 group than the ypT1 group (average, 16.2 vs. 13.0; $p<0.001)$. More patients in the ypT1 group received postoperative therapy than those in the pT1 group $(18 \%$ vs. $1 \%$; $p<0.001)$. No significant differences were observed in other clinical and pathological factors between the 2 groups. 
Table 1. Patient characteristics

\begin{tabular}{|c|c|c|c|}
\hline Characteristics & урT1 $(n=720)$ & pT1 $(n=3,037)$ & $p$ value \\
\hline \multicolumn{4}{|l|}{ Sex, $n(\%)$} \\
\hline Male & $460(64)$ & $1,781(59)$ & \multirow{2}{*}{0.010} \\
\hline Female & $260(36)$ & $1,256(41)$ & \\
\hline Age, mean $\pm S D$, years & $61.2 \pm 0.4$ & $62.3 \pm 0.2$ & 0.030 \\
\hline \multicolumn{4}{|l|}{ Race, $n(\%)$} \\
\hline White & $582(81)$ & $2,490(82)$ & \multirow{3}{*}{$<0.001$} \\
\hline Black & $79(11)$ & $216(7)$ & \\
\hline Others & $59(8)$ & $331(11)$ & \\
\hline \multicolumn{4}{|l|}{ Histological type, $n$ (\%) } \\
\hline Mucinous/signet ring cell & $13(2)$ & $38(1)$ & \multirow[b]{2}{*}{0.207} \\
\hline Others & $707(98)$ & $2,999(99)$ & \\
\hline \multicolumn{4}{|l|}{ Pathological grading, $n$ (\%) } \\
\hline Well/moderate & $545(76)$ & $2,518(83)$ & \multirow{2}{*}{$<0.001$} \\
\hline Others & $175(24)$ & $519(17)$ & \\
\hline \multicolumn{4}{|l|}{ LNs dissected, $n$} \\
\hline Mean \pm SD & $13.0 \pm 0.5$ & $16.2 \pm 0.2$ & $<0.001$ \\
\hline$\geq 12, n(\%)$ & $348(48)$ & $999(33)$ & \multirow{2}{*}{$<0.001$} \\
\hline$<12, n(\%)$ & $372(52)$ & $2,038(67)$ & \\
\hline \multicolumn{4}{|l|}{ Preoperative therapy, $n$ (\%) } \\
\hline Radiotherapy & $49(7)$ & 0 & \multirow{3}{*}{-} \\
\hline Chemotherapy & $19(3)$ & 0 & \\
\hline Chemoradiotherapy & $652(90)$ & 0 & \\
\hline Postoperative chemotherapy & $130(18)$ & $32(1)$ & $<0.001$ \\
\hline
\end{tabular}

p, pathological data; yp, pathological data following preoperative chemoradiotherapy; SD, standard deviation; LN, lymph node.

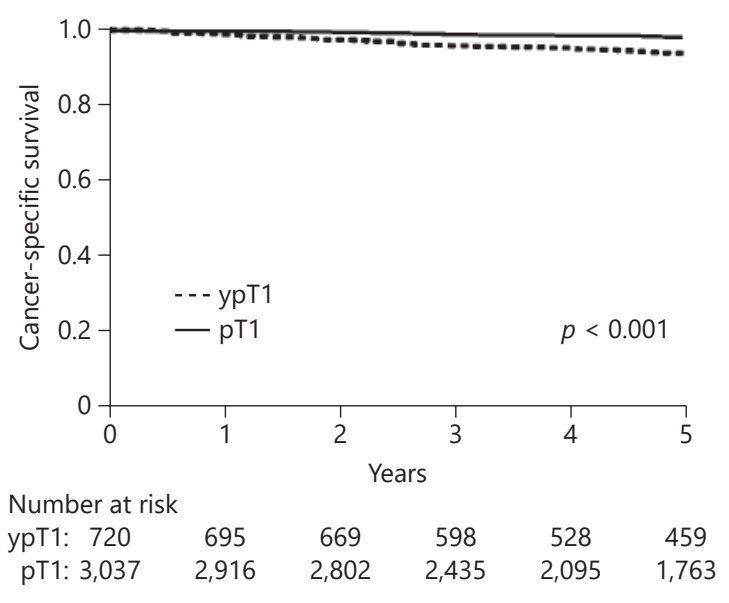

Fig. 1. CSS curves for patients with rectal cancer with pathological T1 stage, stratified by preoperative therapy (ypT1 vs. pT1).

Cancer-Specific Survival and Overall Survival

Stratified by Preoperative Therapy

CSS and OS were compared according to the use of preoperative CRT. Although the CSS for ypT1 patients

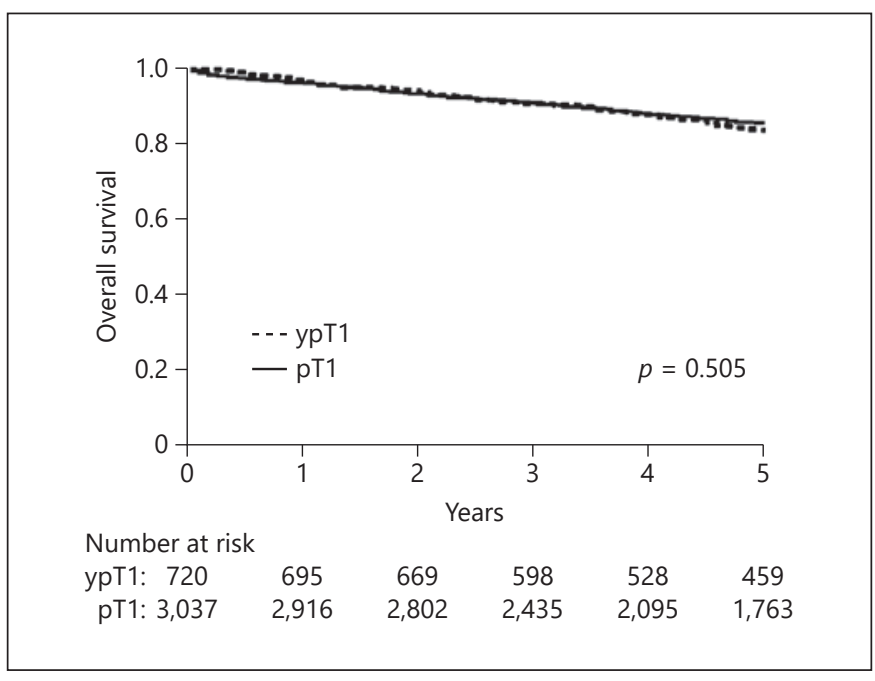

Fig. 2. OS curves for patients with rectal cancer with pathological T1 stage, stratified by preoperative therapy (ypT1 vs. pT1).

was poorer than that for $\mathrm{pT} 1$ patients (5-year CSS rate: $94 \%$ vs. $98 \%, p<0.001$; Fig. 1 ), the OS for ypT1 patients did not differ from that for the $\mathrm{pT} 1$ group (5-year OS rate: $84 \%$ vs. $86 \%, p=0.505$; Fig. 2 ). 
Table 2. Univariate and multivariate analyses of prognostic factors of CSS

\begin{tabular}{|c|c|c|c|c|c|c|}
\hline Characteristics & $H R$ & $95 \% \mathrm{Cl}$ & $p$ value & $H R$ & $95 \% \mathrm{Cl}$ & $p$ value \\
\hline Age ( $\geq 63$ vs. $<63$ years) & 1.13 & $1.07-1.22$ & $<0.001$ & 2.36 & $1.60-3.49$ & $<0.001$ \\
\hline Race (Black vs. others) & 0.99 & $0.85-1.16$ & 0.946 & & & \\
\hline Race (White vs. others) & 1.00 & $0.90-1.11$ & 0.987 & & & \\
\hline LNs dissected, $n(\geq 12$ vs. $<12)$ & 1.43 & $0.48-1.01$ & 0.059 & & & \\
\hline Preoperative therapy (yes vs. no) & 2.95 & $0.87-8.88$ & 0.084 & 2.85 & $0.75-9.09$ & 0.077 \\
\hline Postoperative chemotherapy (yes vs. no) & 2.81 & $1.46-4.84$ & $<0.001$ & 1.56 & $0.84-2.90$ & 0.157 \\
\hline
\end{tabular}

$\mathrm{HR}$, hazard ratio; $\mathrm{Cl}$, confidence interval; LN, lymph node.

Table 3. Univariate and multivariate analyses of prognostic factors of OS

\begin{tabular}{|c|c|c|c|c|c|c|}
\hline Characteristics & $\mathrm{HR}$ & $95 \% \mathrm{Cl}$ & $p$ value & $\mathrm{HR}$ & $95 \% \mathrm{Cl}$ & $p$ value \\
\hline Age ( $\geq 63$ vs. $<63$ years) & 3.33 & $2.85-3.90$ & $<0.001$ & 3.30 & $2.81-3.86$ & $<0.001$ \\
\hline Race (Black vs. others) & 1.96 & $1.38-2.78$ & $<0.001$ & 2.00 & $1.41-2.83$ & $<0.001$ \\
\hline Race (White vs. others) & 1.58 & $1.20-2.10$ & 0.001 & 1.57 & $1.18-2.07$ & 0.002 \\
\hline LNs dissected, $n(\geq 12$ vs. $<12$ ) & 0.75 & $0.65-0.86$ & $<0.001$ & 0.83 & $0.72-0.95$ & 0.009 \\
\hline Preoperative therapy (yes vs. no) & 1.29 & $0.79-2.12$ & 0.313 & & & \\
\hline Postoperative chemotherapy (yes vs. no) & 1.06 & $0.77-1.47$ & 0.711 & & & \\
\hline
\end{tabular}

$\mathrm{HR}$, hazard ratio; $\mathrm{Cl}$, confidence interval; $\mathrm{LN}$, lymph node.

Factors Associated with Cancer-Specific Survival and Overall Survival

To identify prognostic factors associated with CSS and OS, univariate and multivariate analyses were performed. As shown in Table 2, the univariate analysis identified age, histological type, preoperative therapy, and postoperative chemotherapy as significant factors associated with CSS. Multivariate analysis identified old age ( $\geq 63$ years) and histological type (mucinous/signet ring cell) as independent predictors of worse CSS. Preoperative therapy was not independently associated with CSS.

As shown in Table 3, sex, age, race, and number of lymph nodes dissected were significant factors associated with OS using univariate analysis. Multivariate analysis identified male, old age ( $\geq 63$ years), black and white (vs. others), and the number of dissected lymph nodes $(<12)$ as independent predictors of worse OS. Preoperative therapy was not independently associated with OS.

\section{Discussion}

We previously reported on the prognosis of 18 patients with ypT1N0 stage lower rectal cancer in comparison with 68 patients with pT1N0 stage cancer who did not undergo preoperative CRT in our hospital and showed that there was no difference in RFS between ypT1 and pT1 patients; preoperative CRT was not a prognostic factor [14]. In the current study, we have performed a similar analysis using the data from $>3,000 \mathrm{ypT} 1$ or pT1 patients available in the SEER database and showed that (i) 
there was no difference in OS between ypT1 and pT1 rectal cancer patients and (ii) preoperative therapy was not a prognostic factor of CSS or OS. Importantly, these results are consistent with our previous observations in a single-center cohort of 86 patients [14].

In the GLOBOCAN 2018 database from 185 countries, the cumulative mortality rate for rectal cancer was higher in men than in women [15]. In a retrospective epidemiological study that analyzed 34,970 patients with stage I-IV rectal cancer in Germany, 5-year OS rates were higher in women than in men [16]. Another study using the SEER database reported that female patients with stage I-III rectal cancer had longer disease-specific survival and OS in an analysis of 105,511 patients [17]. In the present study, sex was independently associated with poor OS (hazard ratio: 1.21, $p=0.011$ ). Therefore, differences in survival between men and women were also observed in rectal cancer patients even when confined to the pathological T1 stage.

We found that age $\geq 63$ years was independently associated with poor CSS (hazard ratio: $2.36, p<0.001$ ) and OS (hazard ratio: $3.30, p<0.001$ ). A systematic review by the Colorectal Cancer Collaborative Group similarly reported that CSS and OS were lower in elderly patients with colorectal cancer. Furthermore, they speculated that these results may partly be explained by the fact that elderly patients are more likely to present with later-stage disease, were less likely to undergo curative surgery, and had higher postoperative mortality [18].

We found that OS worsened in the following order: black $>$ white $>$ others. These results are consistent with those reported by the American Cancer Society for the period 2013-2017 that rates of colorectal cancer death among black patients $(19.0$ per 100,000$)$ were $40 \%$ higher than those of white patients $(13.8$ per 100,000$)$ and twice as higher than those of others $(9.5$ per 100,000) [19]. Furthermore, they also concluded that these results were associated with insurance status [19].

Regarding histological type and prognosis of rectal cancer, a single-center study that analyzed data from 5,320 patients with rectal cancer showed that the mean survival time among patients with signet ring cell carcinoma was 45.4 months, which was significantly shorter than that of other histological types (78.5 months, $p=$ 0.002 ) [20]. A recent meta-analysis of 8 studies demonstrated that mucinous rectal cancer treated with neoadjuvant CRT was associated with higher rates of a positive resection margin (odds ratio: $0.078, p=0.002$ ) and poor OS (odds ratio: $0.318, p<0.001$ ) [21]. Similarly, we also found that mucinous or signet ring cell carcinoma of $(y)$
pT1 stage was independently associated with poor CSS (hazard ratio: $3.43, p=0.007$ ), which may reflect the aggressiveness of these histological types.

According to the American Society of Clinical Oncology and the European Society for Medical Oncology, the number of lymph nodes dissected $(<12)$ was a risk factor of recurrence in patients with stage II colorectal cancer [22, 23]. Our analysis showed that this parameter was independently associated with poor OS $(p=0.009)$ in (y)pT1 stage. A small number of harvested nodes was also associated with poor CSS with a marginal significance on the univariate analysis. Similarly, a retrospective observational study conducted in 13 hospitals in the Netherlands demonstrated that lymph node yield $\geq 10$ was independently associated with a lower risk of recurrence in patients with pT1 colorectal cancer (hazard ratio $0.20 ; p<0.01$ ) [24]. Collectively, these results underscore the importance of performing appropriate oncologic dissection of the lymph nodes even during surgery for $\mathrm{T} 1$ rectal cancer.

Whether patients with ypT1N0 rectal cancer receive a survival benefit from adjuvant chemotherapy has drawn much attention because a few recent studies stated that adjuvant chemotherapy was associated with improved prognosis even in rectal cancer patients who have achieved a pathological complete response after neoadjuvant CRT $[25,26]$. However, in the current study, postoperative chemotherapy was not a prognostic factor of CSS or OS. Postoperative chemotherapy may not be beneficial for patients with ypT1 or pT1 rectal cancer.

Despite these confirmatory observations, this study contains several limitations. We previously demonstrated that only low tumor location from the anal verge was a significant factor of poor RFS [11]; unfortunately, information on tumor location was unavailable in the SEER database, and we could not address the impact of low tumor location on CSS or OS. Although we excluded patients lacking information on the number of harvested nodes from analyses, this does not necessarily mean that all subjects underwent TME-based surgery because a breakdown of surgical procedures was not available in the SEER database. Moreover, incomplete information on important pathological and clinical parameters such as clinical stage, lymphovascular invasion, perineural invasion, serum tumor markers, performance status, and comorbidity also limited a more extensive analysis of prognostic factors.

To summarize, OS of ypT1 rectal cancer did not differ from that of pT1 rectal cancer by the current analyses that assessed the data of over 3,000 patients. Preoperative therapy was not an independent prognostic factor of CSS and OS.
Taira et al. 


\section{Statement of Ethics}

The study is exempt from ethics committee approval. SEER data are deidentified before release and do not contain any personally identifying information. As the data are publicly available, no ethical approval is required.

\section{Conflict of Interest Statement}

The authors have no conflicts of interest to declare.

\section{Funding Sources}

This research is supported by Grants-in-Aid for Scientific Research (C: Grant No. 19K09114, C: Grant No. 19K09115, C: Grant No. 20K09051, Challenging Research [Exploratory]: Grant No. 20K21626, and B: Grant No. 21H02778) from the Japan Society for the Promotion of Science.

\section{Author Contributions}

T.T. and H.N. contributed to the conception, design, and acquisition of data. K.K., K.S., K.M., S.E., J.K., T.O., Y.Y., S.A., Y.N., H.A., H.S., and S.I. contributed to the analysis and interpretation of data. T.T. and H.N. contributed to drafting the article. K.K., K.S., K.M., S.E., J.K., T.O., Y.Y., S.A., Y.N., H.A., H.S., and S.I. contributed to revising it critically for significant intellectual content. All approved the final version for publication.

\section{Data Availability Statement}

All data generated or analyzed during this study are included in this article. Further enquiries can be directed to the corresponding author.

\section{References}

1 Kawai K, Ishihara S, Nozawa H, Hata K, Kiyomatsu T, Tanaka T, et al. Recent advances in neoadjuvant chemoradiotherapy in locally advanced rectal cancer. J Anus Rectum Colon. 2017 May; 1(2):39-44.

2 Capirci C, Valentini V, Cionini L, De Paoli A, Rodel C, Glynne-Jones R, et al. Prognostic value of pathologic complete response after neoadjuvant therapy in locally advanced rectal cancer: long-term analysis of 566 Ypcr patients. Int J Radiat Oncol Biol Phys. 2008 Sep; 72(1):99-107.

3 Maas M, Nelemans PJ, Valentini V, Das P, Rödel C, Kuo LJ, et al. Long-term outcome in patients with a pathological complete response after chemoradiation for rectal cancer: a pooled analysis of individual patient data. Lancet Oncol. 2010 Sep;11(9):835-44.

4 Rodel C, Martus P, Papadoupolos T, Füzesi L, Klimpfinger M, Fietkau R, et al. Prognostic significance of tumor regression after preoperative chemoradiotherapy for rectal cancer. I Clin Oncol. 2005 Dec;23(34):8688-96.

5 Vecchio FM, Valentini V, Minsky BD, Padula GD, Venkatraman ES, Balducci M, et al. The relationship of pathologic tumor regression grade (TRG) and outcomes after preoperative therapy in rectal cancer. Int J Radiat Oncol Biol Phys. 2005 Jul;62(3):752-60.

6 Deng Y, Chi P, Lan P, Wang L, Chen W, Cui L, et al. Neoadjuvant modified FOLFOX6 with or without radiation versus fluorouracil plus radiation for locally advanced rectal cancer: final results of the Chinese FOWARC trial. J Clin Oncol. 2019 Dec;37(34):3223-33.
7 Conroy T, Bosset JF, Etienne PL, Rio E, François É, Mesgouez-Nebout N, et al. Neoadjuvant chemotherapy with FOLFIRINOX and preoperative chemoradiotherapy for patients with locally advanced rectal cancer (UNICANCER-PRODIGE 23): a multicentre, randomised, open-label, phase 3 trial. Lancet Oncol. 2021 May;22(5):702-15.

8 Bahadoer RR, Dijkstra EA, van Etten B, Marijnen CAM, Putter H, Kranenbarg EM, et al. Short-course radiotherapy followed by chemotherapy before total mesorectal excision (TME) versus preoperative chemoradiotherapy, TME, and optional adjuvant chemotherapy in locally advanced rectal cancer (RAPIDO): a randomised, open-label, phase 3 trial. Lancet Oncol. 2021 Jan;22(1):29-42.

9 Du CZ, Chen YC, Cai Y, Xue WC, Gu J. Oncologic outcomes of primary and post-irradiated early stage rectal cancer: a retrospective cohort study. World J Gastroenterol. 2011 Jul; 17:3229-34.

10 Huh JW, Kim CH, Kim HR, Kim YJ. Oncologic outcomes of pathologic stage I lower rectal cancer with or without preoperative chemoradiotherapy: are they comparable? Surgery. 2011 Nov;150(5):980-4.

11 Li N, Jin J, Yu J, Li S, Tang Y, Ren H, et al. Down-staging depth score to predict outcomes in locally advanced rectal cancer achieving ypI stage after neoadjuvant chemoradiotherapy versus de novo stage $\mathrm{pI}$ cohort: a propensity score-matched analysis. Chin J Cancer Res. 2018 Jun;30(3):373-81.
12 Yeop Oh S, Bae Kim Y, Wook Suh K. Oncologic outcome of ypT1-2N0 rectal cancer after neoadjuvant chemoradiotherapy compared with pT1-2N0 rectal cancer. Am J Clin Oncol. 2017 Oct;40(5):512-6.

13 Wan JF, Zhu J, Li GC, Sun WJ, Zhang Z. YpT1-2N0 rectal cancer after neoadjuvant chemoradiation has lower survival compared with pT1-2N0 rectal cancer. Oncotarget. 2015 Dec;6(38):41056-62.

14 Taira T, Nozawa H, Kawai K, Sasaki K, Murono K, Kaneko M, et al. Oncological outcomes of pathological T1 lower rectal cancer patients with or without preoperative chemoradiotherapy. In Vivo. 2020 Nov;34(6):355964.

15 Bray F, Ferlay J, Soerjomataram I, Siegel RL, Torre LA, Jemal A. Global cancer statistics 2018: GLOBOCAN estimates of incidence and mortality worldwide for 36 cancers in 185 countries. CA Cancer J Clin. 2018 Nov;68(6): 394-424.

16 Berger MD, Yang D, Sunakawa Y, Zhang W, Ning Y, Matsusaka S, et al. Impact of sex, age, and ethnicity/race on the survival of patients with rectal cancer in the United States from 1988 to 2012. Oncotarget. 2016 Aug;7(33): 53668-78.

17 Majek O, Gondos A, Jansen L, Emrich K, Holleczek B, Katalinic A, et al. Sex differences in colorectal cancer survival: population-based analysis of 164,996 colorectal cancer patients in Germany. PLoS One. 2013 Jul;8(7):e68077.

18 Simmonds P, Best L, Baughan C, Buchanan R, Davis C, Fentiman I, et al. Surgery for colorectal cancer in elderly patients: a systematic review. Lancet. 2000 Sep;356(9234):968-74. 
19 Siegel RL, Miller KD, Goding AS, Fedewa SA, Butterly LF, Anderson JC, et al. Colorectal cancer statistics, 2020. CA Cancer J Clin. 2020 Jan;70(1):145-64.

20 Nissan A, Guillem JG, Paty PB, Wong WD, Cohen AM. Signet-ring cell carcinoma of the colon and rectum: a matched control study. Dis Colon Rectum. 1999 Sep;42(9):1176-80.

21 McCawley N, Clancy C, O’Neill BD, Deasy J, McNamara DA, Burke JP. Mucinous rectal adenocarcinoma is associated with a poor response to neoadjuvant chemoradiotherapy: a systematic review and meta-analysis. Dis Colon Rectum. 2016 Dec;59(12):1200-8.
22 Benson AB 3rd, Schrag D, Somerfield MR, Cohen AM, Figueredo AT, Flynn PJ, et al. American Society of Clinical Oncology recommendations on adjuvant chemotherapy for stage II colon cancer. J Clin Oncol. 2004 Aug;22(16):3408-19.

23 Schmoll HJ, Van Cutsem E, Stein A, Valentini V, Glimelius B, Haustermans K, et al. ESMO Consensus Guidelines for management of patients with colon and rectal cancer. A personalized approach to clinical decision making. Ann Oncol. 2012 Oct;23(10):2479-516.

24 Backes Y, Elias SG, Bhoelan BS, Groen JN, van Bergeijk J, Seerden TC, et al. The prognostic value of lymph node yield in the earliest stage of colorectal cancer: a multicenter cohort study. BMC Med. 2017 Dec;15(1):129.
25 Dossa F, Acuna SA, Rickles AS, Berho M, Wexner SD, Quereshy FA, et al. Association between adjuvant chemotherapy and overall survival in patients with rectal cancer and pathological complete response after neoadjuvant chemotherapy and resection. JAMA Oncol. 2018 Jul;4(7):930-7.

26 Turner MC, Keenan JE, Rushing CN, Gulack BC, Nussbaum DP, Benrashid E, et al. Adjuvant chemotherapy improves survival following resection of locally advanced rectal cancer with pathologic complete response. J Gastrointest Surg. 2019 Aug;23(8):1614-22. 